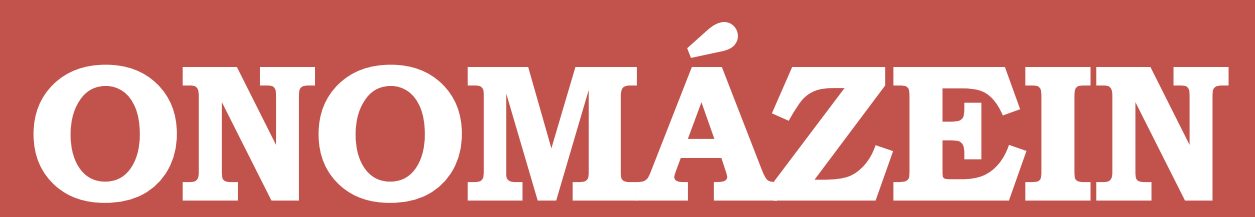

Revista de lingüística, filología y traducción
PONTIFICIA UNIVERSIDAD CATÓLICA DE CHILE FACULTAD DE LETRAS

\title{
La variación en los resúmenes de medicina y lingüística: un análisis desde la organización retórica y la teoría de la valoración
}

Variation in medicine and linguistics abstracts: an analysis based on the rhetorical organization and appraisal theory

\section{Benjamín Cárcamo Morales}

Pontificia Universidad Católica de Valparaíso Chile

\section{(c) $(i)$}

Benjamín Esteban Cárcamo Morales: Instituto de Literatura y Ciencias del Lenguaje, Facultad de Filosofía y Educación, Pontificia Universidad Católica de Valparaíso, Chile. | E-mail: benjamincarcamo.m@gmail.com 


\section{Resumen}

El resumen se ha vuelto un género discursivo fundamental en el mundo académico debido a su papel clave en la diseminación del conocimiento científico. Por esto, se vuelve de interés analizar la manera en la cual este género materializa lingüísticamente sus propósitos en distintas comunidades disciplinares. El presente artículo describe la variación de la organización retórica en las disciplinas de medicina y lingüística y la forma en la cual los autores evalúan la información que presentan en este género discursivo. Para ello, se hace uso de la propuesta de organización retórica del resumen de Hyland (2000) y la dimensión de actitud de la teoría de la valoración de Martin y White (2005). Los resultados de este estudio muestran que las organizaciones P-M-Pr y P-M-Pr-C son las de mayor ocurrencia en ambas disciplinas y que las frecuencias de las movidas de introducción y conclusión son menores en resúmenes de lingüística. En cuanto a la realización del sistema de actitud, ambas disciplinas tienden a privilegiar la apreciación en la introducción y producto. La disciplina de medicina, además, muestra una inclinación a utilizar recursos de apreciación en la movida de conclusión. Estos hallazgos permiten dar cuenta de manera novedosa de la variación disciplinar al evidenciar cómo se configura la subjetividad del autor en la escritura académica del resumen.

Palabras clave: organización retórica; resumen; sistema de actitud; teoría de la valoración; variación disciplinar.

\section{Abstract}

The abstract has become a fundamental genre in the academy due to its role in the dissemination of scientific knowledge. For this reason, it is important to describe this genre in the context of different disciplines. This article describes the rhetorical variation in medicine and linguistics as well as the way in which the information is evaluated in these disciplines. To do this, Hyland's (2000) proposal for rhetorical organization and Martin and White's (2005) appraisal theory are used. The results show that the organizations P-P-Pr and P-M-Pr-C are the most frequent in both disciplines, although in linguistics the moves of introduction and conclusion are less common. Both disciplines privilege appreciation in the introduction and product moves. Medicine, in particular, shows a trend to display appreciation in the conclusion move. These findings help account for variation in a novel manner considering not only rhetorical organization but also evaluation.

Keywords: rhetoric organization; abstract; attitude; appraisal theory; disciplinary variation. 


\section{Introducción}

Pese a que la escritura es una invención humana, esta tiende a ser asumida como un fenómeno tan natural como la oralidad. Una de las razones fundamentales por las cuales esto ocurre es su actual ubicuidad (Navarro, 2014). La escritura es parte esencial tanto de la vida cotidiana como del mundo académico. Esto hace que el aprender a escribir sea una de las tareas más importantes que un individuo enfrenta para insertarse de forma efectiva en la sociedad.

Una de las inserciones sociales más difíciles es la integración a una comunidad disciplinar. Para lograr esto, un individuo debe adquirir las competencias lingüísticas necesarias que le permitan comprender y producir textos característicos de una disciplina particular. Este desafío ha causado el aumento exponencial de programas dedicados a la alfabetización académica (AA) en contextos universitarios (González e Ibáñez, 2017), los cuales enfrentan el desafío de "favorecer el acceso de los estudiantes a las diferentes culturas escritas de las disciplinas" (Carlino, 2013: 310). La manera en la cual cada una de estas disciplinas da forma a las características retóricas, sociales y léxicogramaticales de sus textos de manera particular suele denominarse variación disciplinar (Hyland, 2000).

La variación disciplinar ha recibido especial atención desde los estudios del discurso académico a través del estudio de géneros de formación y géneros expertos (Navarro, 2014). Desde una visión educativa focalizada en el discurso académico, la variación disciplinar ha sido explorada en relación al currículo y metodologías de enseñanza, llevando a conclusiones como la necesidad de motivar la investigación en la disciplina desde pregrado (Lueddeke, 2008). De manera similar, otros estudios han revelado el rol que juega la disciplina en la escritura académica universitaria de los estudiantes, el cual cambia según el programa en el cual se estudia (North, 2005; Hyland, 2008; Hardy y Römer, 2013). Entre estos hallazgos destaca que no existe solo un vocabulario académico, sino que este varía y que, por lo tanto, se puede hablar de escrituras académicas en forma plural. Como resultado, se puede establecer que cada escritura académica plasma las diferentes maneras de ver y pensar el mundo (Hyland, 2008; Durrant, 2017).

Por otra parte, se han investigado corpus de escritura de miembros expertos de la comunidad discursiva, a través de la recopilación de publicaciones de las respectivas comunidades disciplinares, principalmente en revistas de investigación científica (Mur Dueñas, 2007; Pho, 2008; González, 2011; Ibáñez y otros, 2015). Entre los géneros discursivos más analizados se encuentra el resumen debido a su corta extensión, presencia en toda disciplina de carácter científico y creciente importancia en el mundo académico (Bondi y Lorés-Sanz, 2014). Este género ha sido analizado en relación con su variación con respecto a aspectos como las relaciones de coherencia que lo conforman (Ibáñez y otros, 2015), sus movidas retóricas (Kanoksilapatham, 2005; Quintanilla-Espinoza, 2016) y los recursos a través de los que el autor expresa su subjetividad (Pho, 2008; Alonso-Almeida, 2014; Bondi, 2014; Jiang y Hyland, 2016). Esta última 
línea es de especial importancia debido a la necesidad de que el escritor configure su identidad académica y establezca sus argumentos al mismo tiempo que interactúa con las voces de otros escritores (Hood, 2010; Guerra-Lyons y Herrera-Bonillla, 2017).

Una de las principales propuestas para explorar la manera en la cual el autor se posiciona con respecto al conocimiento es la teoría de la valoración (Martin, 2000; Martin y White, 2005; Hood, 2010). Esta propuesta busca describir los recursos semióticos disponibles para la evaluación desde una visión sistémico-funcional del lenguaje. Desde esta óptica, estos recursos se agrupan en 3 sistemas: la actitud (attitude), el compromiso (engagement) y la gradación (graduation) (Martin y Maton, 2017). Estos tres sistemas aluden a la expresión de sentimientos y juicios respecto a lo que se evalúa, al posicionamiento de la voz del autor en relación con otras voces y a la matización o intensificación de los sentimientos expresados, respectivamente.

Las aplicaciones de esta propuesta han revelado que explorar uno de los 3 sistemas puede ya revelar hallazgos interesantes. Por ejemplo, Hood (2011) mostró, analizando el compromiso en su subcategoría de heteroglosia, que las humanidades mostraban prototípicamente una visibilidad mayor en la proyección de sus fuentes que las ciencias naturales. Así, una cláusula como como investigadora feminista, quiero... sería característica de las humanidades, mientras que en las ciencias esto ocurriría principalmente a través de recursos de citación numéricos que ocurren fuera de la cláusula, como en un enfoque para incorporar moléculas dentro del gel de sílice ${ }^{45}$... (Hood, 2011). En cuanto a la variación disciplinar, se ha mostrado que disciplinas como los estudios culturales (EC) tienden a hacer referencia a voces de participantes que son parte del estudio realizado, lo cual realza la visión local del fenómeno estudiado. Por el contrario, disciplinas más científicas como la lingüística aplicada (LA) aluden a las voces de otros investigadores. Las voces de los propios autores en la LA son rara vez utilizadas como fuentes de conocimiento alcanzando apenas un $7 \%$ de todas las instancias, mientras que en los EC esta estrategia ocurre con un 42\% de frecuencia (Hood, 2007).

Tomando en cuenta estas ideas, se puede señalar que deben conocerse las prácticas discursivas de las respectivas comunidades científicas para poder insertarse en estas de manera exitosa. En el caso de la escritura del resumen es necesario saber la manera en la cual esta se configura a nivel retórico y léxico-gramatical según la disciplina, además de las formas aceptadas a través de las cuales el escritor puede plasmar su voz autoral. En este sentido, cobra importancia la exploración en detalle del resumen a la luz de teorías como la de la valoración para revelar los patrones prototípicos de escritura en distintas comunidades disciplinares.

La presente investigación tiene dos objetivos. En primer lugar, busca determinar la variación retórica en el resumen de dos disciplinas representativas de distintas áreas del conocimiento: lingüística y medicina. La primera es usualmente vinculada a las ciencias blandas mientras que la segunda a las ciencias duras (Cavalieri, 2014). Asimismo, el segundo objetivo es examinar la realización del sistema de actitud en ambas disciplinas. El dar cuenta de 
ambos objetivos permitirá caracterizar la variación disciplinar entre las disciplinas, lo cual brindará información empírica que apoye la viabilidad de utilizar de manera complementaria la teoría de la valoración con el análisis de organización retórica. El artículo se divide en las siguientes cuatro secciones: se comienza por revisar la literatura relacionada al resumen y su organización retórica y la teoría de la valoración. Posteriormente, se comparte la metodología utilizada para la investigación. Luego, se muestran los resultados, los cuales son discutidos a la luz de la teoría y otros estudios. Por último, se presentan conclusiones respecto a las implicancias de la investigación llevada a cabo.

\section{Marco teórico}

\subsection{La variación disciplinar en el resumen}

Según Bhatia (2014), los estudios del discurso pueden ser agrupados en tres etapas que coexisten hoy en día: Ia textualización, organización y contextualización. En la etapa de la textualización, el discurso tiende a ser visto como equivalente a un texto con características prototípicas. De esta manera, los investigadores buscan reconocer patrones lingüísticos a nivel clausular analizando corpus generalmente pequeños. Por ejemplo, Bhatia y Swales (1983) identifican la nominalización como unidad de análisis para explorar el discurso legislativo. En la etapa de organización, el discurso se explora a través de la noción de género y práctica profesional, enfatizando conceptos como los de movida retórica y cultura disciplinar (Swales, 1990). Por último, la etapa de la contextualización extiende la noción de discurso a la de práctica social, llevando al investigador a concentrarse en los propósitos comunicativos institucionalizados, los productos discursivos, los procesos que los subyacen y los individuos que participan de estas instancias. En esta última mirada ha crecido una curiosidad investigativa hacia conceptos como la ideología, la identidad del investigador, los conflictos de intereses y la variación disciplinar, entre otros (Hyland, 2000; Bhatia, 2014).

Uno de los contextos de observación de estos fenómenos ha sido el resumen, el cual ha sido propuesto como esencial en el discurso académico, ya que no solo resume los contenidos del artículo (Bhatia, 1993; Ventola, 1994), sino que también persuade al lector de consultar el artículo de investigación (AIC) (Hyland, 2000). Además, el resumen está presente en diversos eventos académicos y científicos que ocurren en diversas comunidades disciplinares, tales como postulaciones a congresos, participación en proyectos de investigación y escritura de AIC. Su clara presencia en el ambiente científico lo hace un recurso valioso para el estudio de la variación disciplinar (Bondi y Lorés-Sanz, 2014).

Los estudios de la variación disciplinar en el resumen siguen los presupuestos de Bhatia (2014) y pueden ser clasificados como de interés local y/o global. Algunos de los hallazgos a nivel local muestran que en la LA existe una preferencia por el uso de la voz activa (79\%) por sobre la pasiva (21\%), mientras que en ingeniería estos recursos se utilizan de forma más 
equitativa, alcanzando un 55\% y 45\% respectivamente (Saboori y Hashemi, 2013). Por otra parte, Ibáñez, Moncada y Santana (2015) han mostrado que la coherencia se configura a través de distintas relaciones en los resúmenes de biología y derecho. Por ejemplo, mientras en biología son más frecuentes las relaciones de causa-efecto y contraste básico, en derecho es más común que la coherencia se articule a través de relaciones de afirmación-argumento y evento-condición.

Desde una mirada global, el resumen se ha caracterizado haciendo uso de distintas organizaciones retóricas a la luz de distintas disciplinas. Así, se han realizado propuestas en base a análisis de movidas retóricas que proponen usualmente entre 4 y 6 movidas como claves en la articulación de un resumen (Bhatia, 1993; Dos Santos, 1996; Hyland, 2000; Lewin, 2010; Swales y Feak, 2012; Weissberg y Buker, 1990). Dentro de las propuestas de 4 movidas retóricas destaca la de Bhatia (1993), quien sugirió la organización IMRD. La primera movida consistiría de una "introducción” (I) donde se presenta la intención del estudio además de objetivos o el problema de investigación. Luego, el "método" (M), el cual corresponde a una exposición de los procedimientos metodológicos. La tercera movida es la de "resultados" $(\mathrm{R})$, que resume los hallazgos del estudio. En la "discusión" (D) se comparten las implicancias de la investigación realizada. En el polo opuesto, se puede mencionar la organización retórica de 6 movidas de Lewin (2010). La propuesta de este autor incluye las siguientes movidas: "relevancia”, "objetivo", "nicho", "método", "resultados” e "implicancias”. A diferencia de la propuesta de Bhatia (1993), esta organización retórica da un mayor énfasis a elementos de la estructura CARS de la introducción de un AIC (Swales, 1990) que pareciesen ser incorporados en el resumen.

La propuesta clásica utilizada en estudios de variación retórica debido al amplio corpus en base al cual fue diseñada es la de Hyland (2000). Esta propuesta postula 5 movidas retóricas a través de las cuales se articula el resumen, las cuales se presentan en la siguiente tabla.

\section{TABLA 1}

Organización retórica del resumen según Hyland (2000)

\begin{tabular}{ll} 
MOVIDA & FUNCIÓN \\
\hline Introducción & Establece el contexto y motiva la investigación o discusión. \\
\hline Propósito & $\begin{array}{l}\text { Indica el propósito, tesis, o hipótesis señalando la intención detrás de la } \\
\text { investigación. }\end{array}$ \\
\hline Método & $\begin{array}{l}\text { Provee información respecto al diseño, procedimientos, suposiciones, } \\
\text { enfoque, datos, etc. }\end{array}$ \\
\hline Producto & $\begin{array}{l}\text { Presenta hallazgos o resultados principales y lo que fue logrado en la } \\
\text { invetigación. }\end{array}$ \\
\hline Conclusión & $\begin{array}{l}\text { Interpeta los resultados llevándolos más allá del alcance de la investigación, } \\
\text { realiza inferencias, apunta hacia aplicaciones o posibles implicaciones. }\end{array}$ \\
\hline
\end{tabular}


La validez de esta propuesta se ha visto solidificada por propuestas como la de Swales y Feak (2012), quienes, desde la noción de movida retórica, también ofrecen 5 movidas para el apartado resumen. Además, esta ha sido utilizada en numerosos estudios de variación retórica (Suntara y Usaha, 2013; Seesaw y Tangkiengsirisin, 2014; Ibáñez y otros, 2015; Ibáñez y Moncada, 2017). Los resultados de estos estudios han revelado que la frecuencia de aparición de algunas de estas movidas está vinculada a la disciplina en la cual se escribe.

Ibáñez y Moncada (2017), al contrastar 80 resúmenes de biología con 80 de historia, señalan que existe una variación respecto a las movidas que cada disciplina prioriza. Por ejemplo, los investigadores notan que la movida de propósito ocurre menos en biología (16,3\%) que en historia (36,3\%). En cambio, la movida de producto está presente en más de un tercio de los resúmenes de biología (77,5\%) y solo en alrededor de la mitad (52,5\%) de los resúmenes de historia. Por su parte, Seesaw y Tangkiengsirisin (2014) realizaron un análisis contrastivo de 200 resúmenes publicados en ciencias ambientales y lingüística aplicada. Los resultados de su trabajo indican que mientras la organización retórica más común en ciencias ambientales resulta ser I-P-M-Pr-C, en el caso de la lingüística aplicada es P-M-Pr-C.

\subsection{Los sistemas de la teoría de la valoración}

La escritura académica requiere de la expresión de subjetividad. Aun cuando esta puede no ser evidente, los escritores deben hacer uso de un lenguaje evaluativo que les permita vincularse tanto con las proposiciones que formulan como con sus potenciales destinatarios (Hood, 2010). Con el fin de estudiar este fenómeno sociodiscursivo se han generado diversas propuestas, tales como la teoría de la evaluación (Hunston y Thompson, 2000), el metadiscurso (Mauranen, 1993; Hyland, 2005; Ädel, 2006) y la teoría de la valoración (Martin, 2000; Martin y White, 2005), entre otras. Esta última ha emergido circunscrita en una de las teorías del lenguaje más relevantes: la lingüística sistémico funcional (LSF).

Desde la LSF se ha reconocido el significado como relacional y paradigmático, lo cual implica que el lenguaje funciona como un recurso semiótico que provee sistemas de elecciones para sus usuarios. De esta manera, las palabras y sus agrupaciones no tienen significado, sino que significan en relación con otras elecciones y contextos particulares (Halliday y Matthiessen, 2014). Desde un modelo relacional de esta naturaleza, la descripción lingüística involucra la manera en la cual los recursos se vinculan y su formalización. Este proceso resulta en explicitar las redes sistémicas que evidencian vinculaciones paradigmáticas a nivel de sistema. La teoría de la valoración, en particular, se puede definir como una propuesta descriptiva de la forma en la cual los significados evaluativos se relacionan unos con otros de manera de construir posiciones evaluativas y posicionamientos intersubjetivos respecto a entidades y proposiciones en el texto (Guerra-Lyons y Herrera-Bonilla, 2017; Martin, 2017). En otras palabras, es un enfoque hacia la exploración del significado interpersonal realizado a través de lenguaje evaluativo desde la mirada de la LSF. En este sentido, cabe señalar que, pese a que 
su nombre incluya la palabra teoría, Martin (2017) ha señalado que la teoría de la valoración no alcanza el nivel de teoría; más bien, es una propuesta de descripción de los recursos evaluativos del sistema de la lengua.

La teoría de la valoración se articula a través de 3 dominios que interactúan: Ios sistemas semánticos de "compromiso", "actitud” y "gradación”. Estos sistemas funcionan al nivel de la semántica del discurso, lo cual implica un espacio de significación entre el género y la léxico-gramática (Hood, 2010). Los 3 dominios de la valoración, a su vez, incluyen sus propias subcategorías divisibles que reflejan elecciones semánticas más delicadas, siguiendo la lógica de redes de sistemas de la LSF (figura 1 ).

\section{FIGURA 1}

Dominios de la teoría de la valoración

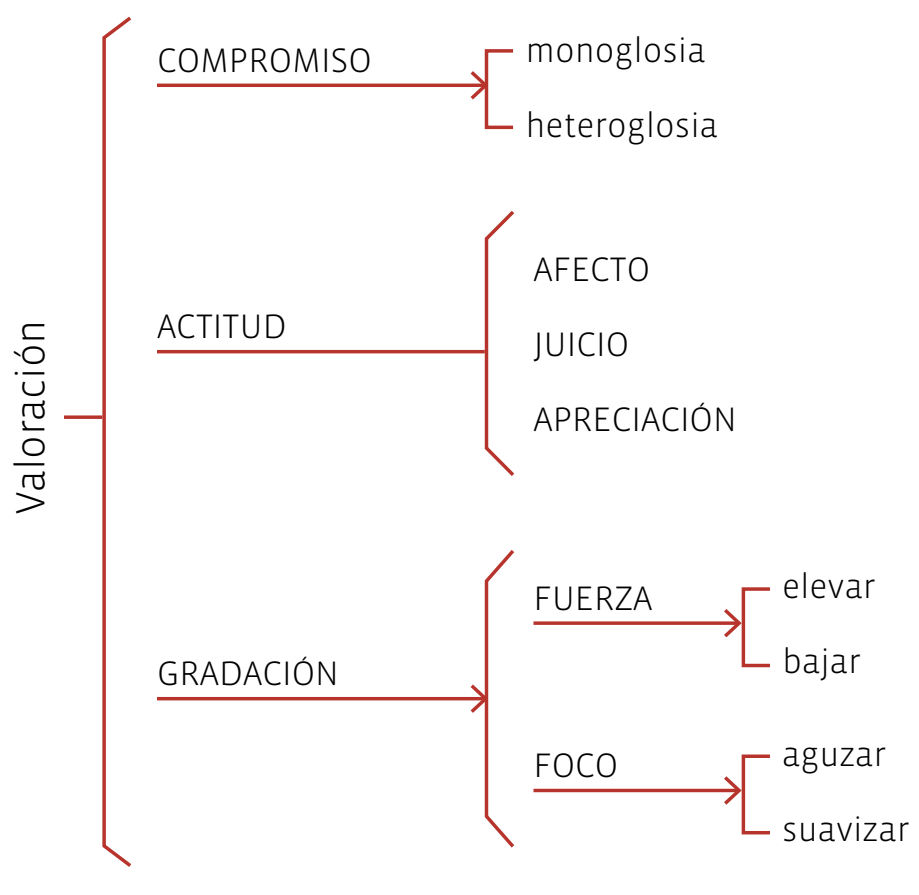

En primer lugar, la gradación se refiere a la manera en la cual matizamos o intensificamos los sentimientos expresados y las clasificaciones de significado a través de los subsistemas de fuerza y foco (Hood, 2005). La fuerza se refiere a la inclusión de valores que permiten modificar la intensidad en el tono de una proposición, a través de, por ejemplo, adverbios de intensificación. Un caso de gradación-fuerza sería el de un enunciado como creo que estás completamente equivocado. El foco, por su parte, permite realizar el potencial de significado de forma vaga a lo largo de un continuo. Por lo tanto, el foco incluiría expresiones que pueden tanto agudizar (este es un concierto de verdad) como atenuar (fui a algo así como un concierto) el significado. 
Por otra parte, el compromiso explora los recursos utilizados para posicionar la voz del hablante en relación con las otras voces que circundan su discurso. Siguiendo las propuestas de Bakhtin, la teoría de la valoración considera que todo acto comunicativo es dialógico en el sentido de que lo que se produce es influenciado o se refiere de una u otra manera a un texto ya producido, al mismo tiempo que anticipa posibles respuestas de lectores u oyentes potenciales (Martin y White, 2005). De esta manera, el sistema semántico del compromiso involucra múltiples categorías, tales como la modalidad, polaridad, evidencialidad, intensificación, atribución y consecuencialidad. Es decir, todos los recursos que permiten que la voz autoral se vincule con otras en un contexto comunicativo. Los dos subsistemas que articulan este sistema son la monoglosia y la heteroglosia. La monoglosia puede ocurrir como una evaluación a nivel de lo "dado por sentado" (taken for granted), ya que en este caso el escritor no reconoce posiciones alternativas respecto a un evento. Por otra parte, la heteroglosia es el sistema que agrupa la manera en la cual se pueden incluir otras voces dentro del texto; las opciones que se asocian a este sistema se resumen a través de la figura 2.

\section{FIGURA 2}

El sistema de compromiso en la heteroglosia (Martin y White, 2005)

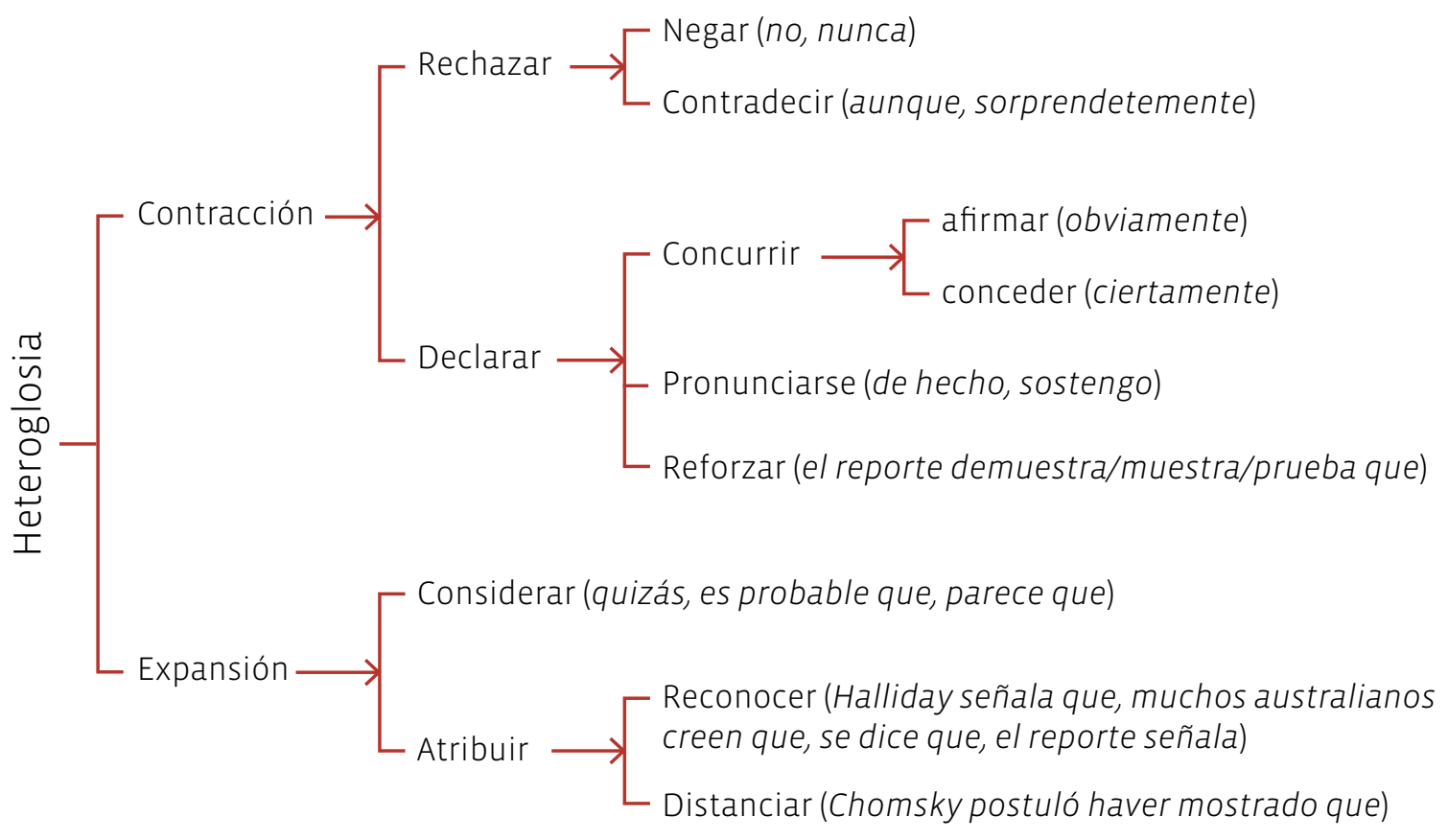

Tal como muestra la figura 2, la heterog losia recoge dos categorías amplias, cuya clasificación depende de su función a nivel intersubjetivo. En el caso de que se permita la validez de una posición y voces alternativas, se considera una expansión dialógica. En cambio, se considera contracción si es que la alternativa que se introduce actúa como una voz que restringe o rechaza esta posibilidad de diálogo. 
Por último, la actitud involucra los aspectos relacionados a los sentimientos, específicamente aquellos recursos de expresión de afecto, juicio y apreciación. El sistema del afecto abarca los recursos que permiten construir y negociar sentimientos, tales como la felicidad, seguridad y satisfacción. Este sistema se puede notar en una cláusula como la última tragedia ha causada un gran pesar. El juicio, en cambio, se preocupa de evaluar comportamientos según normas o principios aceptados por la sociedad. Un ejemplo de este caso sería el siguiente: la dirección del colegio ha sido cruel. Los juicios pueden estar ligados a la estima, la cual suele evidenciarse a través del habla, o a la sanción social, la cual se vincula a documentos escritos. Por su parte, la apreciación no se enfoca en comportamientos, sino que considera los recursos utilizados para construir el valor de las cosas, seres humanos y fenómenos naturales. La apreciación, entonces, puede dividirse en cómo reaccionamos a las cosas, su composición y su valor (Martin y White, 2005). Un ejemplo de apreciación estaría en la frase metodológicamente armonioso. Los tres recursos pueden tener valores tanto positivos como negativos.

Martin y White (2005) sugieren que el juicio y la apreciación podrían ser comprendidos como sentimientos institucionalizados, tal como lo presenta la figura 3.

\section{FIGURA 3}

Juicio y apreciación como afecto institucionalizado (Martin y White, 2005: 45)

Ética/moral (reglas y regulaciones)

Sentimientos institucionalizados como propuestas

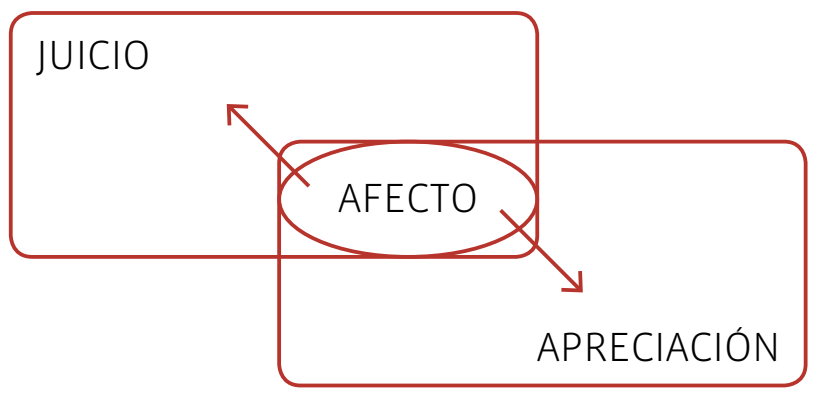

Sentimientos institucionalizados como proposiciones estética/valor (criterios y evaluación)

La figura 3 muestra el juicio y la apreciación como sentimientos institucionalizados. En el caso del juicio, estos sentimientos se verían realizados como propuestas que siguen reglas éticas o morales. En cambio, en el caso de la apreciación, los sentimientos institucionalizados se materializan como juicios de valor o estéticos. El afecto estaría en un punto medio.

Con respecto a sus realizaciones lingüísticas, estas ocurren a través de distintos recursos léxico-gramaticales. La tabla 2 resume algunos de los recursos positivos y negativos empleados para la expresión de afecto, juicio y apreciación. 


\section{TABLA 2}

Realizaciones léxico-gramaticales del sistema de actitud y clasificación

\section{CATEGORÍA EJEMPLOS}

Afecto

a) (In)felicidad: feliz, tristemente, pesar, lloró, detestó, etc.

b) (In)satisfacción: interesado, aburrido, retar, estar absorto, etc.

c) (In)seguridad: intranquilo, asustó, confiado, cómodo, etc.

Juicio 1) Estima social

a) Normalidad (¿cuán especial?): natural, encantador, impredecible, etc.

b) Capacidad (¿cuán capaz?): astuto, competente, torpe, etc.

c) Tenacidad (¿cuán confiable?): heroico, leal, obstinado, cobarde, etc.

2) Sanción social

a) Veracidad (¿cuán honesto?): verdadero, creíble, manipulador, etc.

b) Integridad (¿cuán irreprochable?): moral, altruista, corrupto, etc.

Apreciación $\quad$ a) Reacción-Impacto (atención): cautivador, emocionante, tedioso, etc.

b) Reacción-Calidad (gusto): bueno, encantador, feo, grotesco, etc.

c) Composición-Balance (armonía): simétrico, lógico, distorsionado, etc.

d) Composición-Complejidad (dificultad de seguir): simple, elegante,

impreciso, simplista, etc.

e) Valor (vale la pena): profundo, creativo, convencional, inútil, etc.

Como se puede apreciar en la tabla 2, las expresiones lingüísticas utilizadas para cada uno de los 3 sistemas son a su vez subdivididas bajo distintos criterios que permiten configurar estas dimensiones. En el caso del afecto los recursos se distribuyen en las categorías de felicidad, seguridad y satisfacción. Los juicios se vinculan a estima respecto a la normalidad, capacidad o tenacidad de una acción. Por su parte, la sanción social implica aspectos relacionados a la legalidad y moralidad. Finalmente, los recursos de apreciación se configuran en la reacción que producen en el autor, su opinión respecto a la composición del objeto o fenómeno y el valor.

La teoría de la valoración ha sido ocasionalmente vinculada a otras propuestas que permiten estudiar la manera en la que un escritor expresa su subjetividad en el texto; una de estas es la teoría del metadiscurso (Hyland, 2005). La teoría del metadiscurso considera tanto recursos lingüísticos que guían al lector (interactivos) como aquellos que permiten que el autor adopte una postura frente al texto (interaccionales). Estos últimos recursos se superponen con algunos de los mecanismos incluidos en la teoría de la valoración. De hecho, la teoría de la valoración incluye estos recursos interaccionales como parte de los dominios de la heteroglosia (p. ej., compromiso) y la gradación (p. ej., enfatizadores), pero además considera dentro otros recursos que no encuentran correlatos en la propuesta de Hyland (2005). Entre estos destaca el dominio de la actitud. De esta manera, el análisis desde la teoría de la valoración, en particular desde este último dominio, se vuelve relevante para dar cuenta de fenómenos sociodiscursivos que pueden pasar inadvertidos al hacer uso de otros marcos teóricos.

Además, cabe señalar que la teoría de la valoración, aun cuando suele ser usada de forma independiente, puede complementar un análisis de corpus realizado desde propuestas clási- 
cas como la del análisis de las movidas retóricas (Hood, 2010). El uso conjunto con propuestas que permiten analizar la organización retórica de un género discursivo debiese permitir una descripción acabada de las convenciones de las distintas comunidades discursivas que se deseen estudiar. El presente estudio se propone utilizar una combinación de la teoría de la valoración y el análisis de movidas retóricas con el fin de contrastar la escritura de resúmenes en las disciplinas de lingüística y medicina.

\section{Metodología}

Para realizar el estudio de la variación disciplinar de los resúmenes de medicina y lingüística por medio de la teoría de la valoración, se comenzó por la confección de un corpus de trabajo en base a criterios específicos con el fin de evitar sesgos tales como el idioma, la línea editorial de una revista y los estándares de los textos analizados. Una vez confeccionado el corpus, se analizó la organización retórica de cada uno de los resúmenes haciendo uso de la propuesta de Hyland (2000). Finalmente, para analizar la variación de los recursos evaluativos de los autores se utilizó el sistema de actitud de la teoría de la valoración. A continuación, se detalla cada uno de estos pasos.

\subsection{Corpus}

El corpus de la presente investigación se conformó en base a 60 resúmenes pertenecientes a las disciplinas de lingüística y medicina. Estos resúmenes fueron extraídos de 6 revistas científicas escritas en español indexadas en Web of Science (WoS). Tres revistas científicas eran del área de lingüística y las otras tres de medicina. En el caso de los resúmenes de lingüística, se tomaron 10 resúmenes de las siguientes revistas: Signos, RLA e Ibérica. Los resúmenes de medicina fueron tomados de las revistas Clínica y Salud, Salud Colectiva y Revista de la Asociación Española de Neuropsiquiatría. Al igual que con los resúmenes de lingüística, se tomaron 10 de cada revista con el fin de reducir un posible sesgo asociado a una línea editorial determinada. Todos los resúmenes que conformaron el corpus fueron publicados en los últimos 3 años. El número escogido es similar al de otros estudios que hacen uso de un análisis manual (Pho, 2008; Saboori y Hashemi, 2013; Diani, 2014; Ibáñez y otros, 2015).

\subsection{Procedimiento}

El análisis del corpus siguió una metodología descendente, la cual se caracteriza por la aplicación de una propuesta teórica a un corpus, tal como lo realiza el análisis de movidas clásico de Swales (Biber, Connor y Upton, 2007). Así, en el presente estudio se ha utilizado la propuesta de Hyland (2000) para la examinación del corpus de manera de identificar las movidas presentes en cada resumen y la forma en las cuales estas se articulan formando organizaciones retóricas. De manera similar, para la identificación de los recursos de afecto, juicio y apreciación, se considera la articulación propuesta por Martin y White (2005). 
Pese a que tanto el análisis de movidas retóricas como la teoría de la valoración siguen una metodología descendente, es importante señalar que estas provienen de escuelas distintas. La primera se alinea con la línea investigativa de ESP (English for specific purposes). Por otra parte, la teoría de la valoración es representativa de la escuela de la LSF (lingüística sistémico funcional). Desde un punto de vista metodológico, se espera que estas perspectivas se complementen de manera tal de tener una mirada más abarcadora del fenómeno de la variación, al verse este nutrido por una teoría del lenguaje como la de la LSF. Con respecto a esto, Hood (2011: 9) señala que "estudios de género de la ESP ofrecen una perspectiva orientada al texto, la cual es compatible en muchos niveles con un modelamiento teórico de la LSF”. En este sentido, se espera que la discusión de los datos haga dialogar la importancia de la comunidad discursiva (Swales, 1990) con la teoría del lenguaje que ofrece la LSF. El etiquetado del corpus tanto para las movidas retóricas como para el análisis de la valoración fue hecho por dos anotadores. En ambos casos tuvieron un alto índice de acuerdo alcanzando un $k=.86$ en movidas retóricas y $k=.81$ en la valoración respectivamente.

Para el análisis de las movidas, se suele recomendar la utilización de frecuencias para discriminar entre las que son opcionales y las obligatorias (Swales, 1990). En esta presente investigación se sigue la propuesta de Kanoksilapatham (2015), quien sugiere 3 distintos tipos de movida: obligatoria, convencional y opcional. En el caso de que una movida esté presente en un $\mathbf{1 0 0 \%}$ del corpus se le denomina obligatoria. Las movidas convencionales, por otra parte, serían aquellas que se presentan en más de un 60\% del corpus. Finalmente, aquellas que se presentan en menos de un $60 \%$ se les denomina opcionales. Por su parte, los resultados respecto a recursos de actitud se resumen en una tabla de contingencia, la cual permite contrastar la frecuencia de los recursos del sistema de actitud en las disciplinas estudiadas considerando las movidas retóricas identificadas en el resumen.

\section{Resultados y discusión}

En este apartado se presentan los resultados del análisis en dos secciones distintas. En una primera sección, se comparten la frecuencia de las distintas movidas en el corpus analizado y las organizaciones retóricas más prototípicas de cada disciplina. Luego, se presenta el análisis del sistema de actitud en el corpus y se presentan ejemplos que representan a ambas disciplinas con el fin de evidenciar la variación disciplinar.

\subsection{Organización retórica del resumen en lingüística y medicina}

Como se mencionó con anterioridad, se ha utilizado el marco analítico de Hyland (2000) para identificar las movidas retóricas presentes en cada una de las disciplinas analizadas. El gráfico a continuación muestra los resultados para lingüística. 


\section{FIGURA 4}

Frecuencia de movidas en lingüística

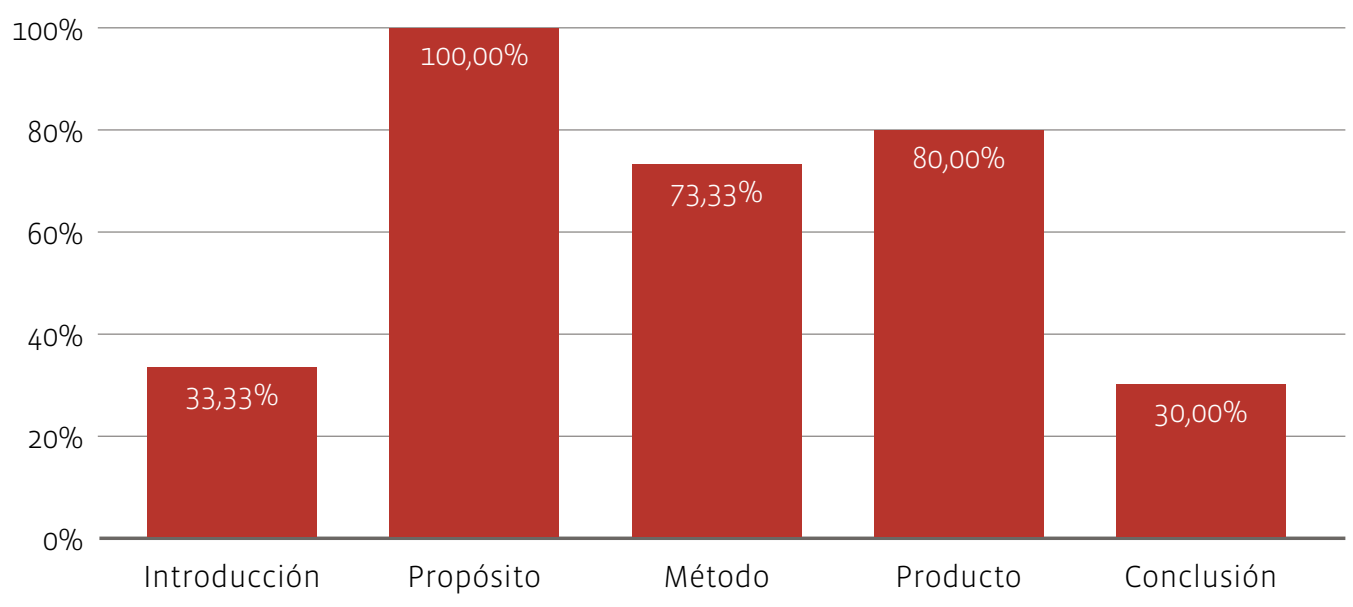

Como se observa en la figura 4, las 5 movidas se encuentran presentes en los resúmenes de lingüística. No obstante, siguiendo el criterio de Kanoksilaphatam (2015) estas son de distinta naturaleza. En cuanto a las movidas opcionales, se pueden identificar las movidas de introducción y conclusión, las cuales alcanzan un 33,33\% y 30\% respectivamente. En el caso de las movidas convencionales se pueden hallar dos: la de método con un 73,33\% y la de producto con un $80 \%$. Por último, existe una movida de carácter obligatorio, ya que ocurre en todos los resúmenes analizados en esta disciplina: la movida de propósito.

Estos resultados son similares en algunos aspectos a los hallazgos de otros investigadores. La movida que se ha identificado como obligatoria, también ha presentado un nivel alto de frecuencia en otros estudios (Hyland, 2000; Pho, 2013; Seesaw y Tangkiengsirin, 2014; Diani, 2014). Por ejemplo, Diani (2014), al analizar contrastivamente resúmenes de lingüística escritos en italiano e inglés, descubrió que la movida de propósito ocurría en un 94,11\% y un 97,87\% respectivamente. De manera similar, Seesaw y Tangkiengsirisin (2014), a través de un análisis contrastivo de resúmenes de lingüística y ciencias ambientales, mostraron que los resúmenes de lingüística contaban en un 94\% con la presencia de esta movida. Por otra parte, la frecuencia de las movidas de método y producto se asemeja a los resultados de estudios como los de Seesaw y Tangkiengsirisin (2014) y Pho (2013), quienes analizaron resúmenes de lingüística y lingüística aplicada en inglés. Estos autores encontraron la movida de método en un $91 \%$ de los resúmenes de lingüística y en un 100\% de los de lingüística aplicada. Asimismo, la de producto se presentaba en un $95 \%$ y en un $100 \%$ del corpus respectivamente.

Respecto a las movidas de introducción y conclusión, estas se presentan en frecuencias bajas. Pese a que Hyland (2000) recalca la importancia de la movida de introducción en las humanidades, en el corpus analizado esta aparece en menos de un tercio de los resúmenes. En efecto, esta frecuencia es menor a la que suele ser hallada en otros estudios donde es cer- 
cana al 50\% (Diani, 2014; Pho, 2013; Seesaw y Tangkiengsirisin, 2014). Ahora bien, cabe señalar que esta movida ha sido poco característica de los corpus en español. Por ejemplo, Lorés-Sanz (2016), en una investigación sobre la variación en resúmenes en sociología escritos en inglés y español, indica que la movida de conclusión suele ser omitida en los resúmenes escritos en español aun cuando esta sea exigida por las revistas en las cuales se publica. Martín-Martín (2003) señala que la movida de conclusión del resumen de lingüística en español aparece solo en un 58\% de los resúmenes de su corpus. Esta baja presencia de la movida coincide con los hallazgos de este estudio.

En el caso de los resúmenes en medicina, la frecuencia de las movidas es presentada en la figura 5.

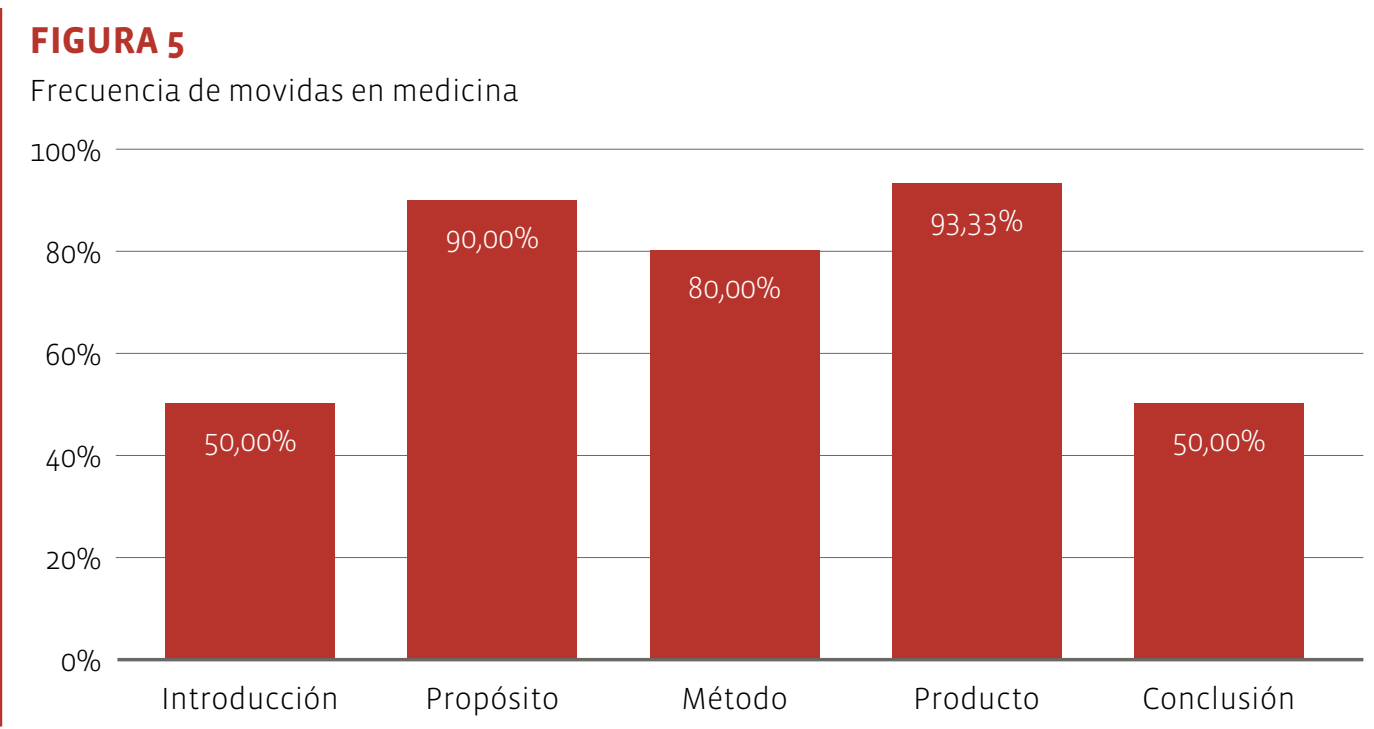

La figura 5 muestra un patrón similar al de lingüística en algunos aspectos. Por ejemplo, pese a que las movidas de conclusión e introducción presentan una frecuencia mayor a la que obtienen en lingüística, ambas son opcionales en ambas disciplinas. Asimismo, las movidas de método y producto serían convencionales, ya que superan ampliamente el 60\%, al igual que en lingüística. La diferencia principal yace en que la movida de propósito es convencional en medicina, pero en lingüística es obligatoria. Además, todas las movidas, exceptuando la de propósito, se presentan con mayor frecuencia en medicina que en lingüística, lo cual indica una mayor complejidad retórica.

Respecto a los resúmenes de medicina, Cavalieri (2014) señala que su presentación suele ser distinta a la de otras disciplinas, ya que la mayoría de las revistas explicita con encabezados cada movida del resumen. Pese a que los resúmenes extraídos para este análisis no presentaban esta característica editorial, se puede conjeturar que, al ser parte de la comunidad discursiva de medicina, los escritores deben estar familiarizados con la expectativa de que se incluyan 
todas estas movidas. De hecho, estudios de corpus en el resumen de medicina han mostrado frecuencias cercanas al 100\% en todas las movidas (Talebinezhad y otros, 2012; Cavalieri, 2014).

Ahora bien, aun cuando se suelen postular organizaciones retóricas con 5 movidas como la de Hyland (2000), la ocurrencia simultánea de las 5 movidas suele ser escasa (Bondi y LorésSanz, 2014). En virtud de esto, las distintas disciplinas suelen mostrar organizaciones retóricas prototípicas que combinan distintas movidas retóricas, tales como I-P-Pr (introducción, propósito y producto) o M-Pr-C (método, producto y conclusión). Las frecuencias de las organizaciones retóricas del resumen en el caso de lingüística se presentan en la figura 6.

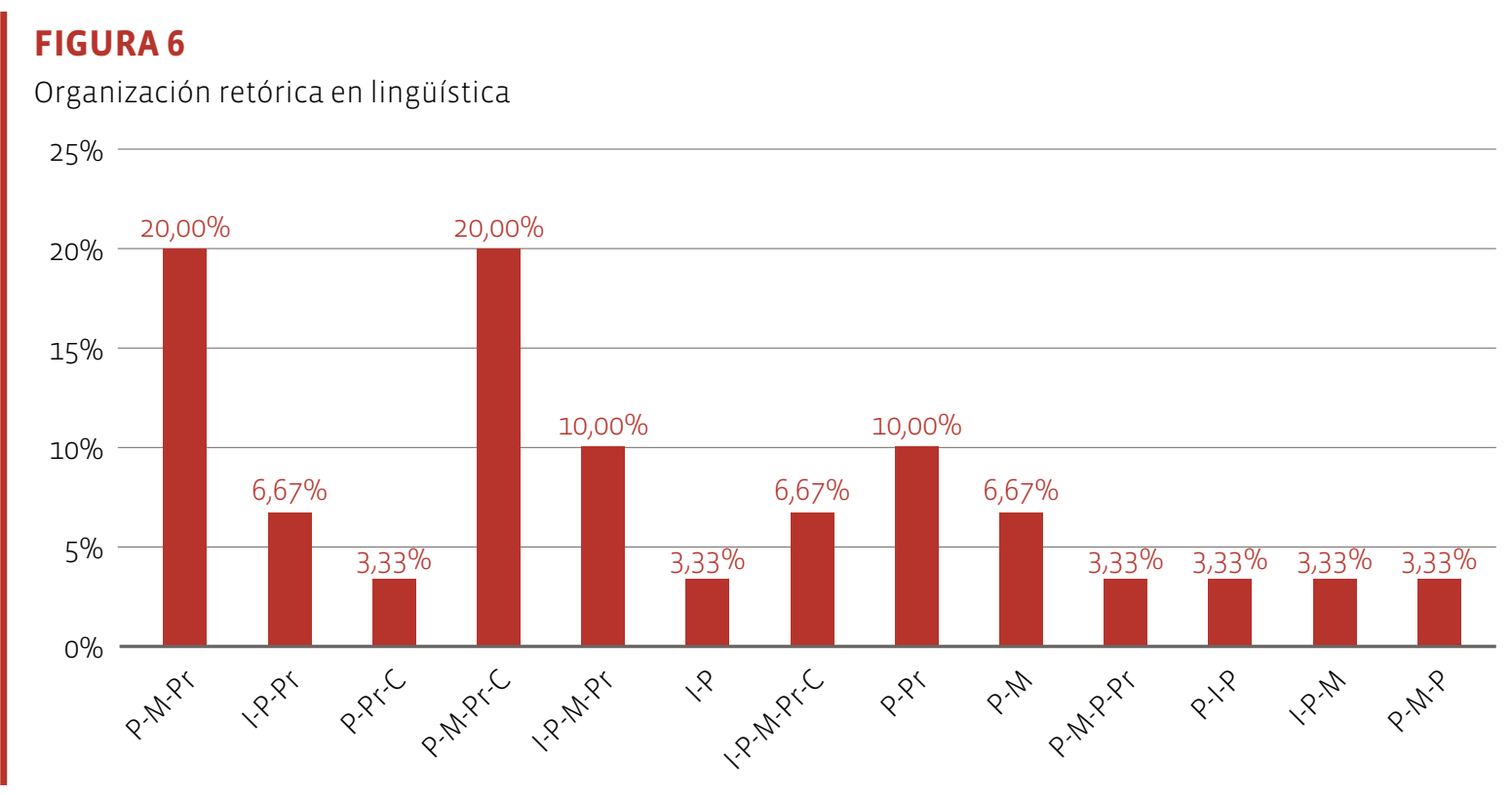

La figura anterior muestra las 13 organizaciones retóricas identificadas en el corpus. Uno de los hallazgos que este gráfico presenta es que las dos organizaciones retóricas de mayor frecuencia son las de P-M-Pry P-M-Pr-C, ambas alcanzando un 20\% de frecuencia. Por otra parte, la organización retórica que incluye las 5 movidas (I-P-M-Pr-C) solo ocurre en un 6,67\% del corpus.

Con respecto a medicina, la organización retórica muestra una variación levemente menor, ya que solo se han identificado 11 opciones, las cuales se presentan en la figura 7.

El gráfico presenta las frecuencias de las organizaciones retóricas de medicina dando cuenta de las articulaciones de movidas retóricas más y menos prototípicas. En el caso de la organización que incluye todas las movidas (I-P-M-Pr-C), esta se presenta con una frecuencia de $13,33 \%$, la cual es mayor que en lingüística, donde solo alcanza un 6,67\%. Las dos organizaciones retóricas con más alta ocurrencia son la de P-M-Pr (16,67\%) y la de P-M-Pr-C (26,67\%). Claramente la mayoría de las organizaciones retóricas en medicina contienen 4 movidas retóricas (54,54\%) en contraste con lo que sucede en lingüística (23,07\%). 


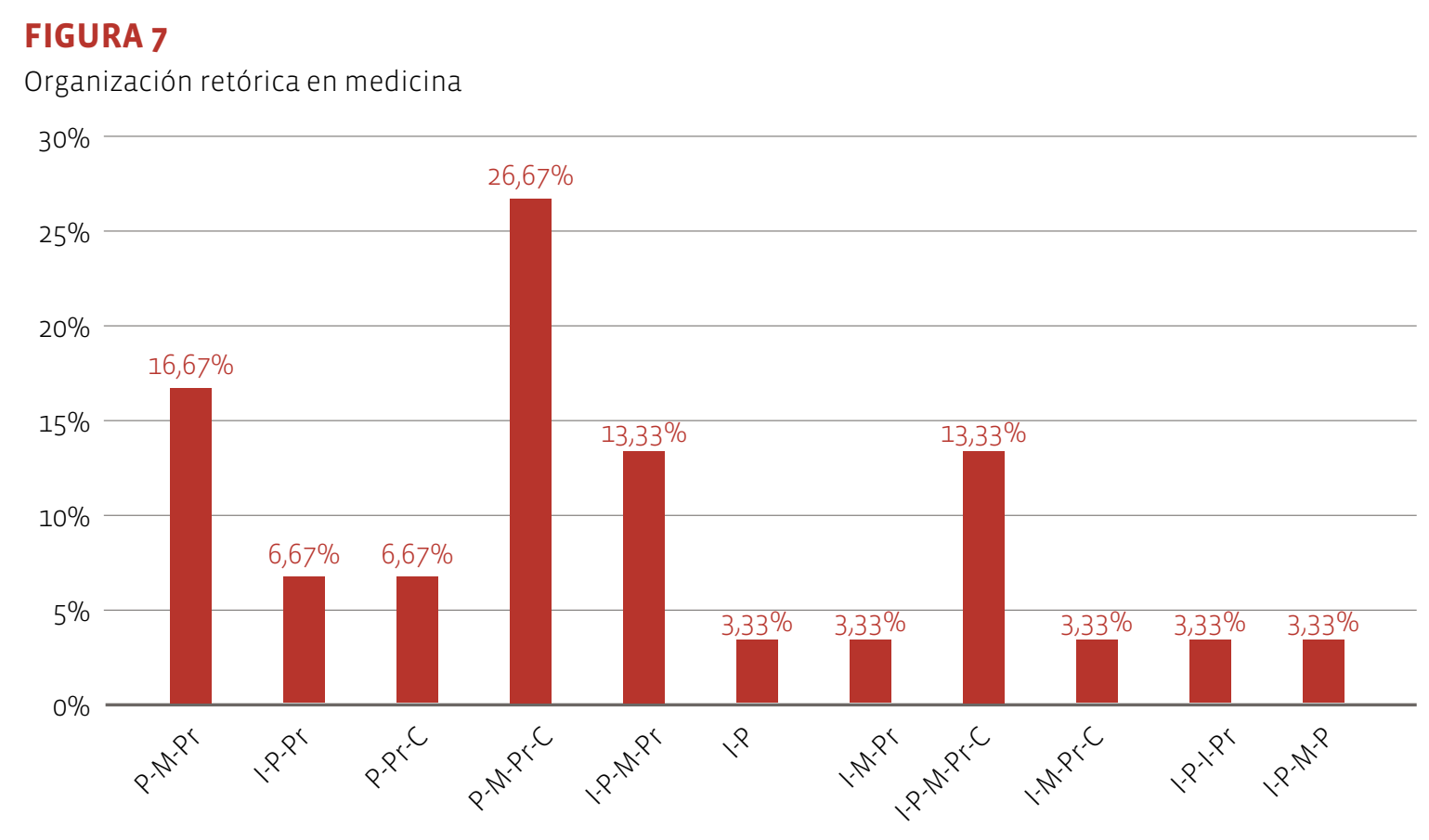

Se puede señalar que no es extraño que dentro de las organizaciones retóricas prototípicas de ambas disciplinas se encuentre la organización P-M-Pr. Martín-Martín (2003) menciona que los resúmenes de lingüística en español se diferencian de aquellos escritos en inglés por configurarse de organizaciones retóricas más simples, es decir, con menos movidas retóricas.

En un análisis contrastivo entre lingüística aplicada y medicina en inglés, Cavalieri (2014), luego de analizar 60 resúmenes de cada disciplina, identificó que la medicina tendía a presentar una organización retórica más compleja. En contraste con la lingüística aplicada, los resúmenes de medicina incluían la movida introductoria del resumen como obligatoria. La autora indica que la importancia de esta movida se debe al foco empírico de la medicina. Por medio de esta movida se puede proveer una descripción del contexto en el cual se realiza el estudio con el propósito de caracterizar los casos o enfermedades estudiadas. Esta movida, según Cavalieri (2014), sería más relevante que otras, como la movida de método. En el caso del corpus analizado, sin embargo, las organizaciones retóricas más prototípicas en medicina carecen en su mayoría de la movida introductoria. Este resultado muestra una mayor coherencia con los hallazgos vinculados a la escritura de resúmenes en español que con la disciplina misma en la cual se escribe.

\subsection{El sistema de actitud en los resúmenes de lingüística y medicina}

A través del análisis del sistema de actitud, se puede notar la manera en la cual el investigador evidencia su subjetividad a través de recursos léxico-gramaticales que le permiten realizar 
comentarios evaluativos. La tabla 3 presenta la frecuencia de recursos para expresar actitud, en sus dimensiones de apreciación, afecto y juicio, destacando si es que este es positivo o negativo. Además, estos se vinculan con las movidas retóricas en las cuales se presentan (introducción, propósito, método, producto y conclusión). Así, la notación “Aprec-Prod(+)" indicaría comentarios evaluativos de apreciación en la movida de producto.

\section{TABLA 3}

El afecto en los resúmenes según movida retórica

\begin{tabular}{|c|c|c|c|c|c|}
\hline CATEGORİA & LING & $\mathrm{FI}(\%)$ & CATEGORÍA & LING & $\mathrm{FI}(\%)$ \\
\hline Aprec-Prod(+) & 12 & $42,86 \%$ & Aprec-Prod(+) & 11 & $32,35 \%$ \\
\hline Aprec-Intr(+) & 7 & $25,00 \%$ & Aprec-Intr(+) & 7 & $20,59 \%$ \\
\hline Aprec-Intr(-) & 3 & $10,71 \%$ & Aprec-Concl(+) & 5 & $14,71 \%$ \\
\hline Aprec-Met(+) & 2 & $7,14 \%$ & Aprec-Intr(-) & 2 & $5,88 \%$ \\
\hline Aprec-Prod(-) & 1 & $3,57 \%$ & Aprec-Met(+) & 2 & $5,88 \%$ \\
\hline Aprec-Prop(+) & 1 & $3,57 \%$ & Afecto-Prod(-) & 2 & $5,88 \%$ \\
\hline Aprec-Concl(+) & 1 & $3,57 \%$ & Aprec-Prod(-) & 1 & $2,94 \%$ \\
\hline Aprec-Prod(-) & 1 & $3,57 \%$ & Aprec-Prop(+) & 1 & $2,94 \%$ \\
\hline Aprec-Concl(-) & 0 & $0,00 \%$ & Aprec-Concl(-) & 1 & $2,94 \%$ \\
\hline Afecto-Prod(+) & 0 & $0,00 \%$ & Afecto-Prod(+) & 1 & $2,94 \%$ \\
\hline \multirow[t]{2}{*}{ Juicio-Prod(+) } & 0 & $0,00 \%$ & Juicio-Prod(+) & 1 & $2,94 \%$ \\
\hline & 28 & $100 \%$ & & 34 & $100 \%$ \\
\hline
\end{tabular}

Como se aprecia en la tabla, ambas disciplinas presentan el uso de recursos de actitud en frecuencias similares. La primera mayoría en ambos casos es la apreciación positiva en la movida de producto. En este caso el autor utiliza la apreciación para establecer que los hallazgos presentan un gran valor para el área disciplinar en la cual escribe.

(1) Este cúmulo de datos se analizó mediante la técnica de análisis de contenido, lo que finalmente se plasma en sendos modelos explicativos en que se visualizan los hitos... (apreciación (+) / lingüística)

(2) Además, puede ser útil en la evaluación de la calidad asistencial que reciben los cuidadores de pacientes esquizofrénicos. (apreciación (+) / medicina)

Una segunda mayoría es la apreciación positiva en la movida retórica de introducción. Utilizando este recurso el autor puede realzar la importancia del tema que abordará en su estudio, de manera de entregar credibilidad al mostrar conocimiento de su comunidad discursiva y 
el rol que el tema estudiado tiene en esta (Hyland, 2000). Esto se evidencia en los siguientes ejemplos de las disciplinas contrastadas.

(3) La guía de viajes se configura como uno de los géneros discursivos más específicos del turismo. (apreciación (+) / lingüística)

(4) La aceptación y respeto de las normas que rigen la sociedad y la unidad familiar son pilares esenciales para la elaboración de un programa terapéutico en personas con trastornos por dependencia de sustancias. (apreciación (+) / medicina)

Aquellas apreciaciones que funcionan de forma negativa en la movida retórica de introducción sirven el mismo propósito. De esta manera, se evalúa el objeto de estudio o la temática explorada como una que no ha sido lo suficientemente investigada hasta entonces.

(5) El feedback correctivo (FC) escrito ha sido un tema polémico en adquisición de segundas lenguas principalmente por los cuestionamientos acerca de su efectividad (Truscott, 1996). (apreciación (-) / lingüística)

(6) Pese al gran avance experimentado por la psicofarmacología, hay preguntas obvias en la práctica cotidiana que no suelen centrar la atención de los investigadores. (apreciación $(-) /$ medicina)

Considerando que la movida de introducción es más escasa en el resumen de lingüística $(33,3 \%)$ en contraste con el de medicina (50\%), es importante destacar que, pese a su frecuencia similar, en el caso de lingüística el uso de apreciación en la introducción ocurre en un 100\% de los casos. Es decir, cada vez que la movida de introducción se presenta en un resumen en lingüística, esta es utilizada para valorar el área u objeto de estudio asociado a la investigación.

Otra diferencia que se desprende del análisis es lo que ocurre en la movida de conclusión. Mientras que la movida de conclusión es utilizada para valorar en solo uno de los nueve $(11,1 \%)$ resúmenes de lingüística que incluyeron la movida, en el caso de medicina esto ocurre en seis de los quince (40\%) que presentan la movida. Esto demuestra un interés mayor en el área de medicina por resaltar las implicancias del estudio en la disciplina y valorar estas contribuciones. De esta forma, en medicina se promueve más evidentemente la relevancia del estudio, lo cual incrementa el valor persuasivo del resumen (Hyland, 2000). Además, el énfasis en la valoración tanto en la introducción como en la conclusión se puede interpretar como muestra de la importancia del foco empírico de los estudios en esta comunidad discursiva (Cavalieri, 2014). Es decir, al ser estos estudios empíricos generalmente centrados en casos, es probable que se haga necesario intentar revelar la manera en la cual estos estudios se enmarcan en un contexto mayor sobre el cual la investigación tiene un alcance claro, tal como muestra el extracto 7. 
(7) La información obtenida es indispensable para el adecuado manejo del flúor y la mejora de la salud pública a través de políticas sanitarias. (apreciación (+) / medicina)

Los recursos de afecto y juicio solo son utilizados marginalmente y se vinculan a investigaciones muy específicas. Por ejemplo, en el caso de lingüística, se encontró un recurso de afecto negativo relacionado a inseguridad, el cual se vinculaba a una sensación de tensión. Asimismo, en salud estos recursos tampoco se muestran con una frecuencia significativa y también ocurren solamente en casos muy específicos. Por lo tanto, no se puede determinar un uso prototípico de estos recursos en el corpus analizado.

En base a este análisis se pueden evidenciar patrones prosódicos en la escritura del resumen a nivel general. Los patrones prosódicos son entendidos como la manera en la cual el significado interpersonal emerge a través de cláusulas y fases del discurso (Martin, 1992; Hood, 2006). En el caso de los resúmenes analizados se visualiza una oscilación en el uso de recursos interpersonales entre lo que se puede caracterizar como movidas subjetivas y movidas objetivas. En primer lugar, se aprecia que la movida de introducción incorpora recursos de apreciación positiva del objeto de estudio o nicho a investigar o negativa con respecto a falencias o limitaciones de estudios previos. Este hallazgo es similar al hecho por Hood (2006) en el análisis de introducciones de artículos de investigación científica. Una vez realizado esto, las movidas de propósito y método se caracterizan por ser altamente objetivas y desprenderse de recursos valorativos. Luego, la movida de producto vuelve a establecer un vínculo entre escritor y lector respecto a la importancia del trabajo escrito, ya que se resalta valorativamente la importancia de los hallazgos del estudio. De hecho, es en esta movida en la cual se concentra la mayor cantidad de recursos valorativos de apreciación. Por último, la movida de conclusión es utilizada solamente en medicina con una alta presencia de apreciación positiva, lo cual demuestra una conciencia mayor respecto a las proyecciones que tiene el estudio en la disciplina.

En resumen, los resúmenes de lingüística y medicina muestran tanto similitudes como diferencias. En cuanto a las similitudes, cabe destacar que existe una necesidad por valorar tanto el campo de estudio en la movida de introducción como los hallazgos en la movida de producto mediante recursos de apreciación principalmente positiva. Esto permite construir dos argumentos concatenados de manera eficaz, los cuales contribuyen al propósito del género discursivo. En primer lugar, se construye el argumento de que el objeto de estudio vale la pena de ser explorado y en segundo lugar el que los hallazgos del estudio son significativos. En cuanto a la variación en el uso de estos recursos, se aprecia que la disciplina de medicina hace uso de recursos valorativos en la movida de conclusión de manera prototípica.

\section{Conclusiones}

La presente investigación descriptiva de corpus ha buscado determinar la variación disciplinar a nivel retórico y del sistema de actitud en los resúmenes de medicina y lingüística. 
Estos objetivos se han alcanzado aplicando dos marcos metodológicos utilizados de forma complementaria pese a pertenecer a distintas escuelas de estudios del género: la propuesta de organización retórica de Hyland (2000) y la teoría de la valoración (Martin y White, 2005).

Los resultados de esta investigación muestran que existe una variación retórica entre ambas disciplinas. Por una parte, los resúmenes de lingüística cuentan con una movida obligatoria, la de propósito; dos movidas convencionales, las de método y producto, y dos opcionales, las de introducción y conclusión. Por otra parte, los resúmenes de medicina siguen la misma tendencia, pero la movida de propósito es convencional y las movidas de método, producto, conclusión e introducción se presentan con una frecuencia mayor que en lingüística. Esto resulta en una mayor complejidad retórica en medicina, en la cual un 54,54\% de las organizaciones retóricas consiste en 4 movidas, mientras este es el caso de solo un 23,07\% de los resúmenes de lingüística. Pese a esto, ambas disciplinas presentan las mismas dos organizaciones retóricas prototípicas: P-M-Pr-C y P-M-Pr. Otro hallazgo de esta investigación es la manera en la cual el sistema de actitud es utilizado de manera general, en el resumen, y de manera más específica en cada una de estas disciplinas. El sistema de actitud tiende a ser utilizado de manera casi exclusiva a través de su dimensión de apreciación. La apreciación ocurre principalmente en las movidas de introducción y producto. Además, en el caso de medicina, hay una preocupación más notoria por evaluar el estudio en el marco de la disciplina a través de la movida de conclusión.

Entre los desafíos para estudios futuros se pueden mencionar dos. En primer lugar, se debe continuar explorando la evaluación en los resúmenes utilizando la teoría de la valoración, en concreto, generando más hallazgos vinculados al sistema de actitud y, además, haciendo uso de sistemas de gradación y compromiso. Esto permitirá tener una visión más holística de la manera en la cual los escritores evalúan en el contexto de la escritura académica. En segundo lugar, se postula que un buen camino a seguir es la realización de un estudio como el aquí presentado con resúmenes en inglés. De esta manera, se podría evidenciar si tanto las similitudes como las diferencias aquí halladas están vinculadas no solo a la disciplina, sino también a la lengua en la cual se escribe.

En síntesis, el análisis a través de la teoría de la valoración ha permitido dar cuenta del funcionamiento de los resúmenes en el nivel de la semántica del discurso. Es decir, ha permitido extender la exploración de este género discursivo al nivel del significado interpersonal en vez de centrarse, como se suele hacer desde otras miradas, en un plano exclusivamente léxico-gramatical a nivel ideacional. Al complementar esta propuesta con la de la organización retórica, se ha podido vislumbrar de manera más holística la forma en la cual el autor produce este género realizando sus funciones descriptiva y persuasiva. Tomando en cuenta la creciente importancia del género resumen en el mundo de la investigación académica, se espera que este estudio permita seguir profundizando en la manera en la cual se expresa la evaluación en textos académicos y su vinculación con la variación disciplinar. 


\section{Bibliografía citada}

ÄDEL,Annelie, 2006: Metadiscourse in LI and L2 English,Amsterdam/Philadelphia:John Benjamins.

Alonso-Almeida, Francisco, 2014: "Evidential and epistemic devices in English and Spanish medical. Computing and legal scientific abstracts: a contrastive study" en Marina Bond y Rosa LoRÉs-SAnz (eds.): Abstracts in academic discourse: Variation and change, Bern: Peter Lang, 21-42.

Bhatia, Vijay, y John Swales, 1983: "An approach to the linguistic study of the legal documents", Fachsprache 5 (3), 98-108.

BhatiA, Vijay, 1993: Analyzing genre: language use in professional settings, Londres: Longman.

BhatiA, Vijay, 2014 [2004]: Worlds of written discourse: A genre-based view, segunda edición, Londres / Nueva York: Bloomsbury Academic.

Biber, Douglas, Ulla Connor y Thomas Upton (eds.), 2007: Discourse on the move. Using corpus analysis to describe discourse structure, Amsterdam: Benjamins.

Bond, Marina, 2014: "Changing voices: Authorial voice in abstracts" en Marina Bond y Rosa LoRés-SAnz (eds.): Abstracts in academic discourse: Variation and change, Bern: Peter Lang, 243-270.

Bond, Marina, y Rosa Lorés-Sanz (eds.), 2014: Abstracts in academic discourse: Variation and change, Bern: Peter Lang.

CarLino, Paula, 2013: “Alfabetización académica diez años después”, Revista Mexicana de Investigación Educativa 18 (57), 355-381.

Cavalierl, Silvia, 2014: "Variation across disciplines. The case of applied linguistics and medicine" en Marina Bond y Rosa Lorés-SAnz (eds.): Abstracts in academic discourse: Variation and change, Bern: Peter Lang, 161-174.

DIANI, Giuliana, 2014: "On English and Italian research article abstracts: Genre variation across cultures" en Marina Bond y Rosa Lorés-Sanz (eds.): Abstracts in academic discourse: Variation and change, Bern: Peter Lang, 65-84.

Dos Santos, Mauro, 1996: "The textual organization of research paper abstracts in applied linguistics", Text 16 (4), 481-499.

DURRANT, Philip, 2017: "Lexical bundles and disciplinary variation in university students' writing: Mapping the territories", Applied Linguistics 38 (2), 165-193. 
González, Cristian, 2011: "La formulación de los objetivos en artículos de investigación científica en cuatro disciplinar: historia, lingüística, literatura y biología”, Linguagem em (Dis)curso $11(2), 401-429$.

González, Cristian, y Romualdo IbÃ̃ez, 2017: "Leer y escribir en contextos académicos" en Romualdo IbAÑez y Cristian GonzÁlez (eds.): Alfabetización disciplinar en la formación inicial docente: Leer y escribir para aprender, Valparaíso: Ediciones Universitarias de Valparaíso, 27-41.

Guerra-Lyons, Jesús, y Marggie Herrera-Bonilla, 2017: “El compromiso en la teoría de la valoración: Conceptos y aplicaciones pedagógicas", Forma y Función 30 (2), 51-69.

Halliday, Michael, y Christian MatThiessen, 2014 [1985]: Halliday's Introduction to Functional Grammar, cuarta edición, Londres: Routledge.

HARDY, Jack, y Ute RömeR, 2013: "Revealing disciplinary variation in student writing: a multi-dimensional analysis of the Michigan Corpus of Upper-level Student Papers (MICUSP)", Corpora $8(2), 183-207$.

Hood, Susan, 2005: "Invocación de actitudes: El juego de la gradación de la valoración en el discurso", Revista Signos 38 (58), 195-220.

Hood, Susan, 2006: "The persuasive power of prosodies: Radiating values in academic writing”, Journal of English for Academic Purposes 5, 37-49.

Hood, Susan, 2007: "Arguing in and across disciplinary boundaries: legitimizing strategies in applied linguistics and cultural studies" en Anne McCaBe, Mick O'Donnell y Rachel WhitTaker (eds.): Advances in language \& education, Londres: Continuum, 185-200.

Hood, Susan, 2010: Appraising research: Evaluation in academic writing, Nueva York: Palgrave Macmillan.

Hood, Susan, 2011: "Writing discipline: Comparing inscriptions of knowledge and knowers in academic writing" en Frances Christie y Karl Maton (eds.): Disciplinarity: Functional linguistics and sociological perspectives, Londres: Continuum International Group, 106-128.

Hunston, Susan, y Geoffrey Thompson (eds), 2000: Evaluation in text: Authorial stance and the construction of discourse, Oxford: Oxford University Press.

HyLAND, Ken, 2000: Disciplinary discourses: Social interactions in academic writing, Londres: Longman.

HyLAND, Ken, 2005: Metadiscourse: Exploring interaction in writing, Londres: Continuum. 
HyLAND, Ken, 2008. "As can be seen: Lexical bundles and disciplinary variation”, Specific Purpo$\operatorname{ses} 27,4-21$.

Ibañez, Romualdo, Fernando Moncada y Andrea Santana, 2015: "Organización retórica y relaciones de coherencia en el abstract de biología”, Estudios Filológicos 56, 51-84.

IbẪ̃ez, Romualdo, y Fernando MoncadA, 2017: “El género resumen en dos disciplinas: una comparación a partir de su organización retórica y relaciones de coherencia” en Romualdo IBÁNez y Cristian GonzÁlez (eds.): Alfabetización disciplinar en la formación inicial docente: Leer y escribir para aprender, Valparaíso: Ediciones Universitarias de Valparaíso, 85-115.

JIANG, Feng, y Ken HyLAND, 2016: "Metadiscursive noun: Interaction and cohesion in abstract moves", English for Specific Purposes 46, 1-14.

Kanoksilapatham, Budsaba, 2005: "Rhetorical structure of biochemistry research articles”, English for Specific Purposes 24 (3), 269-292.

Kanoksilapatham, Budsaba, 2015: "Distinguishing textual features characterizing structural variation in research articles across three engineering sub-discipline corpora", English for Specific Purposes 37, 74-86.

LEwIN, Beverly, 2010: Writing readable research: A guide for students of social science, Londres: Equinox.

LoRÉs-SAnz, Rosa, 2016: "When the local becomes international. The lexicogrammar of rhetorical moves in English and Spanish Sociology abstracts", Languages in Contrast 16 (1), 133-158.

LuEDDEKE, George, 2008. "Reconciling research, teaching and scholarship in higher education: an examination of disciplinary variation, the curriculum and learning", International Journal for the Scholarship of Teaching and Learning 2 (1), 1-17.

MARTIN, Jim, 1992: English text, Amsterdam: John Benjamins.

Martin, Jim, 2000: "Beyond exchange: APPRAISAL systems in English" en Susan Hunston y Geoffrey Thompson (eds.): Evaluation in text: Authorial stance and the construction of discourse, Oxford: Oxford University Press, 142-175.

Martin, Jim, 2017: "The discourse semantics of attitudinal relations: Continuing the study of lexis", Russian Journal of Linguistics 21 (1), 22-47.

MARTIN, Jim, y Peter White, 2005: The language of evaluation: Appraisal in English, Londres / Nueva York: Palgrave/Macmillan. 
MARTIN, Jim, y Karl MATON, 2017: "Systemic functional linguistics and Legitimation Code Theory on education: Rethinking field and knowledge structure", Onomázein, número especial II: LSF y TCL sobre educación y conocimiento, 12-45.

Martín-Martín, Pedro, 2003: "A genre analysis of English and Spanish research paper abstracts in experimental social sciences", English for Specific Purposes 22, 25-43.

Mauranen, Anna, 1993: Cultural differences in academic rhetoric: A textlingusitic study, Frankfurt: Peter Lang.

Mur Dueñas, Pilar, 2007: “I/we focus on....: A cross-cultural analysis of self-mentions in business management research articles", Journal of English for Academic Purposes 6 (2), 143-162.

Navarro, Federico, 2014: "Géneros discursivos e ingreso a las culturas disciplinares: Aportes para una didáctica de la lectura y la escritura en educación superior" en Federico NavarRo (coord.): Manual de escritura para carreras de humanidades, Buenos Aires: Editorial de la Facultad de Filosofía y Letras Universidad de Buenos Aires, 29-52.

NORTH, Sarah, 2005: "Disciplinary variation in the use of theme and undergraduate essays", Applied Linguistics 26 (3), 431-452.

PHo, Phuong, 2008: "Research article abstracts in Applied Linguistics and Educational Technology: A study of linguistic realizations of rhetorical structure and authorial stance”, Discourse Studies 10 (2), 231-250.

Pнo, Phuong, 2008, 2013: Authorial stance in research articles: Examples from applied linguistics and educational technology, Nueva York: Palgrave Macmillan.

Quintanilla-Espinoza, Angie, 2016: “La organización retórica del resumen o abstract del artículo de investigación en educación”, Estudios Pedagógicos XLLII (2), 285-298.

SABOORI, Fahimeh, y Mohammad HASHEMI, 2013: "A cross-disciplinary move analysis of research article abstracts", International Journal of Language Learning and Applied Linguistics World 4, 483-496.

SeESAW, Supachai, y Supong TANGKIENGSIRISIN, 2014: "Rhetorical variation across research article abstracts in environmental science and applied linguistics", English Language Teaching 7 (8), 81-93.

SUnTARA, Watinee, y Siriluck UsAHA, 2013: "Research article abstracts in two related disciplines: Rhetorical variation between linguistics and applied linguistics", English Language Teaching 6 (2), 84-99. 
SwaLEs, John, 1990: Genre analysis. English in academic and research settings, Cambridge: Cambridge University Press.

SWALES, John, y Christine FEAK, 2012 [1994]: Academic writing for graduate students: a course for nonnative speakers of English, tercera edición, Michigan: University of Michigan Press.

Talebinezhad, Mohammad, Zahra Arbabi, Saeed Takı y Mahmood AkHLAghI, 2012: "Rhetorical variation in medical article abstracts written in English and Persian", Journal of Teaching Language Skill 3 (4), 127-144.

Ventola, Eija, 1994: "Abstracts as an object of linguistic study" en Svëtla ČmejRkova, Frantisek DaneŠ y Eva Havlova (eds.): Writing vs. Speaking, Tübingen: Gunter Narr, 333-352.

WeISSBERG, Robert, y Suzanne BukER, 1990: Writing up research. Experimental research report writing for students of English, Nueva Jersey: Prentice Hall Regents. 